PROCEEDINGS OF THE

AMERICAN MATHEMATICAL SOCIETY

Volume 135, Number 5, May 2007, Pages 1505-1510

S 0002-9939(06)08640-0

Article electronically published on November 29, 2006

\title{
A MARKOV-TYPE INEQUALITY FOR ARBITRARY PLANE CONTINUA
}

\author{
ALEXANDRE EREMENKO
}

(Communicated by Juha M. Heinonen)

ABStRaCt. Markov's inequality is

$$
\sup _{[-1,1]}\left|f^{\prime}\right| \leq(\operatorname{deg} f)^{2} \sup _{[-1,1]}|f|,
$$

for all polynomials $f$. We prove a precise version of this inequality with an arbitrary continuum in the complex plane instead of the interval $[-1,1]$.

Theorem 1. Let $E$ be a continuum in the complex plane, and $f$ a polynomial of degree $d$. Then

$$
\operatorname{cap} E \sup _{E}\left|f^{\prime}\right| \leq 2^{1 / d-1} d^{2} \sup _{E}|f| .
$$

Here cap denotes the transfinite diameter (capacity) of a set [2, Ch. 2]. Using the well-known inequality $\operatorname{diam} E \leq 4 \operatorname{cap} E$ we obtain

Corollary. With the assumptions of Theorem 1 we have

$$
\operatorname{diam} E \sup _{E}\left|f^{\prime}\right| \leq 2^{1 / d+1} d^{2} \sup _{E}|f| .
$$

This inequality looks more elementary than (11), but we could not find a direct proof.

To compare these results with Markov's inequality [7, 8], we take $E=[-1,1]$. Then cap $E=1 / 2$, and $\operatorname{diam} E=2$, so (11) and (2) become

$$
\sup _{[-1,1]}\left|f^{\prime}\right| \leq 2^{1 / d} d^{2} \sup _{[-1,1]}|f|,
$$

while Markov's inequality is

$$
\sup _{[-1,1]}\left|f^{\prime}\right| \leq d^{2} \sup _{[-1,1]}|f| .
$$

Thus the estimate of Theorem 1 for $E=[-1,1]$ is asymptotically exact as $d \rightarrow \infty$.

Both (11) and Markov's inequality are precise, and in both of them equality is achieved on the Chebyshev polynomials, but to obtain equality in (11) one has to take $E$ to be the level set of the Chebyshev polynomial, $E=\left\{z:\left|T_{d}(z)\right| \leq 1\right\}$, rather than the interval $[-1,1]$.

Inequality (2) for $E=[-1,1]$ is worse than Markov's inequality by the factor $2^{1 / d}$, and one can show that equality never takes place in (2).

Received by the editors September 1, 2005 and, in revised form, January 3, 2006.

2000 Mathematics Subject Classification. Primary 41A17, 26 D05.

The author was supported by NSF grants DMS-0100512 and DMS-0244421. 
Pommerenke [10] proved a weaker form of (11):

$$
\operatorname{cap} E \sup _{E}\left|f^{\prime}\right| \leq\left(e d^{2} / 2\right) \sup _{E}|f| .
$$

A special case of (11) is the following result conjectured by Erdôs and proved in [5].

Theorem A. Let $f$ be a monic polynomial such that the level set $E(f)=\{z$ : $|f(z)| \leq 1\}$ is connected. Then

$$
\sup _{E(f)}\left|f^{\prime}\right| \leq 2^{1 / d-1} d^{2} .
$$

Equality in Theorem A occurs if and only if $f(z)=c^{-d} T_{d}\left(2^{1 / d-1} c z+b\right)$, where $|c|=1$, and $T_{d}$ is the Chebyshev polynomial defined by

$$
\cos d z=T_{d}(\cos z) .
$$

Theorem A will be used in the proof of the general result. Theorem 1 evidently follows from

Theorem 2. Let $f$ be a polynomial of degree $d$, and $E$ a component of the set $E(f)=\{z:|f(z)| \leq 1\}$. Then

$$
\operatorname{cap} E \sup _{E}\left|f^{\prime}\right| \leq 2^{1 / d-1} d^{2} .
$$

This estimate is best possible, and the equality holds if and only if $f(z)=$ $a T_{d}(c z+b)$ with $c \neq 0,|a|=1$, and $E=E(f)$.

The proof of Theorem 2 is based on the idea which was used in [4, 6] to prove other polynomial inequalities. The method 11 consists of including $f$ in a holomorphic family of polynomials parametrized by critical values and proving that the quantities of interest are subharmonic functions of parameters. Then it remains to check the inequality on the boundary of the parameter domain.

Following [6], we begin by establishing existence of extremal polynomials. Then we will show that for every monic extremal polynomial, the set $E(f)$ is connected, thus reducing Theorem 2 to Theorem A.

Lemma 1. For every positive integer $d$ there exists a polynomial $f^{*}$ of degree at most $d$ and a component $E^{*}$ of the set $E\left(f^{*}\right)$ such that

$$
\operatorname{cap} E^{*} \sup _{E^{*}}\left|\left(f^{*}\right)^{\prime}\right| \geq \operatorname{cap} E \sup _{E}\left|f^{\prime}\right|,
$$

for every polynomial $f$ of degree at most $d$ and every component $E$ of $E(f)$.

Proof. Let $\left(f_{n}\right)$ and $\left(E_{n}\right)$ be extremizing sequences. Notice that the product cap $E_{n} \sup _{E_{n}}\left|f_{n}^{\prime}\right|$ does not change if the independent variable $z$ is replaced by $a z+b$, where $a \neq 0$. So we can use the normalization

$$
\text { cap } E_{n}=1 \text { and } \quad 0 \in E_{n} .
$$

This implies cap $E\left(f_{n}\right) \geq 1$, so

$$
f_{n}(z)=a_{n} z^{d}+\ldots, \quad \text { where }\left|a_{n}\right| \leq 1 .
$$

Let $g_{n}$ be the Green function of the complement of $E_{n}$ with the pole at infinity. We have the Riesz representation

$$
g_{n}(z)=\int_{\mathbf{C}} \log |z-\zeta| d \mu_{n}(\zeta),
$$

\footnotetext{
${ }^{1}$ A method is a device which you used twice 9 .
} 
with some probability measures $\mu_{n}$ whose supports belong to $E_{n}$. Any continuum of capacity 1 has diameter at most 4, 2], so our normalization conditions (5) imply that the supports of $\mu_{n}$ are contained in the disc $D(4)=\{z:|z| \leq 4\}$. Thus one can choose a subsequence such that $\mu_{n} \rightarrow \mu$, where $\mu$ is a probability measure on $D(4)$. Then we have $g_{n} \rightarrow g$ uniformly in the region $\Delta(5)=\{z:|z|>5\}$, so $g_{n}(z) \leq 2 g(z), z \in \Delta(5)$, and by the Principle of Harmonic Majorant, $\left|f_{n}(z)\right|<$ $\exp (2 d g(z)), z \in \Delta(5)$, where we used $\left|a_{n}\right| \leq 1$ from (6). Thus the sequence of our polynomials is uniformly bounded on compact subsets of the plane and so, after choosing a subsequence, we obtain a limit polynomial $f^{*}$. Now (4) is evident. This completes the proof of the lemma.

We continue the proof of Theorem 2. For fixed $d$, we will call $\left(f^{*}, E^{*}\right)$ an extremal pair, if $\operatorname{deg} f^{*} \leq d, E^{*}$ is a component of $E\left(f^{*}\right)$ and (4) is satisfied.

To simplify our notation, let $(f, E)$ be an extremal pair. If the set $E(f)$ is connected, that is, $E=E(f)$, then we can normalize as in (5), and the polynomial $f$ will be monic because cap $E(f)=1$. So Theorem A applies and (3) follows. Now let us assume that the set $E(f)$ is disconnected.

This assumption implies that there is a critical value $a$ of $f$ such that $|a|>1$. Let $D$ be a disc centered at the point $a$ of radius $8 \delta=|a|-1$, so that it does not intersect the unit disc. Let $\chi$ be a continuously differentiable function in the complex plane $\mathbf{C}$, with support in $D$, such that $\chi(a)=1$ and $|\nabla \chi|<1 /(2 \delta)$. An example of such a function is $\left(1-|z-a|^{2} /\left(64 \delta^{2}\right)\right)^{2}$. Let $\Delta$ be the disc of radius $\delta$ centered at 0 . Then for $\lambda \in \Delta$, the map

$$
\psi_{\lambda}=\mathrm{id}+\lambda \cdot \chi
$$

is a quasiconformal homeomorphism of the plane, holomorphic outside $D$ and holomorphically depending on $\lambda$. Now we construct a quasiconformal homeomorphism $\psi_{\lambda}$ which makes the function

$$
f_{\lambda}=\psi_{\lambda} \circ f \circ \phi_{\lambda}^{-1}
$$

analytic in the whole plane. To achieve this, $\phi_{\lambda}$ has to satisfy a Beltrami equation (see [1, Ch. 1C]):

$$
\frac{\partial \phi_{\lambda}}{\partial \bar{z}}=\mu_{\lambda} \frac{\partial \phi_{\lambda}}{\partial z}
$$

where the Beltrami coefficient

$$
\mu_{\lambda}=\left(\mu_{\psi_{\lambda}} \circ f\right) \frac{\overline{f^{\prime}}}{f^{\prime}} \quad \text { and } \quad \mu_{\psi_{\lambda}}=\frac{\partial \psi_{\lambda}}{\partial \bar{w}}: \frac{\partial \psi_{\lambda}}{\partial w} .
$$

Differentiation of (7) with respect to $\bar{\lambda}$ and application of the Beltrami equation shows that $f_{\lambda}$ is analytic. Notice that $\mu_{\psi_{\lambda}}$ and thus $\mu_{\lambda}$ depend analytically on $\lambda$. The existence theorem for the Beltrami equation (also known as the "Measurable Riemann Theorem", 2, Ch. 2, thms. 4, 5]) says that there exists a unique homeomorphic solution $\phi_{\lambda}: \mathbf{C} \rightarrow \mathbf{C}$ normalized by $\phi_{\lambda}(0)=0$ and $\phi_{\lambda}(1)=1$. Moreover, this normalized solution depends analytically on $\lambda$.

As $f_{\lambda}$ defined in (7) is analytic in $\mathbf{C}$, and both $\psi_{\lambda}$ and $\phi_{\lambda}$ are homeomorphisms, we conclude that the $f_{\lambda}$ are polynomials of the same degree as $f$.

Now we claim that $f_{\lambda}$ depends holomorphically on $\lambda$. More precisely,

$$
(z, \lambda) \mapsto f_{\lambda}(z), \quad \mathbf{C} \times \Delta \rightarrow \mathbf{C}
$$


is a holomorphic function of two variables. To verify this, we apply the operator $\partial / \partial \bar{\lambda}$ to both sides of the equation

$$
f_{\lambda} \circ \phi_{\lambda}=\psi_{\lambda} \circ f .
$$

As $\psi_{\lambda}$ is holomorphic in $\lambda$, we obtain

$$
\frac{\partial f_{\lambda}}{\partial \bar{\lambda}} \circ \phi_{\lambda}=-\left(\frac{\partial f_{\lambda}}{\partial z} \circ \phi_{\lambda}\right) \frac{\partial \phi_{\lambda}}{\partial \bar{\lambda}}=0
$$

the last equality holds because $\phi_{\lambda}$ depends holomorphically on $\lambda$. This proves that the map (8) is holomorphic.

Notice that the $z \mapsto \phi_{\lambda}(z)$ are holomorphic on $E$. This is because the $\psi_{\lambda}$ are holomorphic in $\mathbf{C} \backslash D$ and the full preimage $f^{-1}(D)$ is disjoint from $E$ by our choice of $D$.

The polynomials $f_{\lambda}$ are obtained from $f$ by moving the critical value $a$ of $f$ to the point $a+\lambda$.

We put $E_{\lambda}=\phi_{\lambda}(E)$. Then $E_{\lambda}$ is a component of the set $E\left(f_{\lambda}\right)$. Such families of sets $E_{\lambda}$ parametrized by a complex parameter $\lambda$ are called holomorphic motions; they were much studied recently (see, for example, [3]), but we don't use any deep properties of holomorphic motions here. The following two lemmas are almost evident:

Lemma 2. The function $\lambda \mapsto \log \sup _{E_{\lambda}}\left|f_{\lambda}^{\prime}\right|$ is subharmonic.

Proof. In some neighborhood $V$ of $E$ we have

$$
f_{\lambda} \circ \phi_{\lambda}=f \text {. }
$$

Differentiation with respect to $z$ for $z \in V$ gives

$$
\left(f_{\lambda}^{\prime} \circ \phi_{\lambda}(z)\right) \phi_{\lambda}^{\prime}(z)=f^{\prime}(z), \quad z \in E,
$$

where we used the fact that $\phi_{\lambda}$ is holomorphic on $E$. It follows from (9) that the functions $\lambda \mapsto f_{\lambda}^{\prime} \circ \phi_{\lambda}(z)$ are holomorphic for every fixed $z \in V$. So $f_{\lambda}^{\prime} \circ \phi_{\lambda}$ is a holomorphic function of two variables, $\lambda$ and $z$, for $z$ in $V$ and sufficiently small $\lambda$. Taking the logarithm of the modulus and then taking the supremum with respect to $z \in E$ we arrive at the conclusion of the lemma.

Lemma 3. The function $\lambda \mapsto \log \operatorname{cap} E_{\lambda}$ is subharmonic.

This should be well known, and the proof below applies to arbitrary holomorphic motion of a compact set, but we could not find a convenient reference.

Proof. Let

$$
d_{n}(\lambda)=\sup \prod_{1 \leq i<j \leq n}\left|\phi_{\lambda}\left(z_{i}\right)-\phi_{\lambda}\left(z_{j}\right)\right|^{2 / n(n-1)},
$$

where the supremum is taken over all subsets $\left\{z_{j}\right\}$ of $E$ of cardinality $n$. Then $\log d_{n}(\lambda)$ is a subharmonic function. Now, $\left(d_{n}\right)$ is a decreasing sequence [2], and the transfinite diameter can be defined by the formula

$$
\operatorname{cap} E_{\lambda}=\lim _{n \rightarrow \infty} d_{n}
$$

which completes the proof. 
So the function

$$
F(\lambda)=\log \left(\operatorname{cap} E_{\lambda} \sup _{E_{\lambda}}\left|f_{\lambda}^{\prime}\right|\right)
$$

is subharmonic. As the pair $(f, E)$ is assumed to be extremal, we obtain from the Maximum Principle that

$$
F=\text { const. }
$$

To summarize, we proved that if $(f, E)$ is an extremal pair, and $f$ has a critical value $a$ outside of the unit disc, then for every $\lambda \in \Delta$, all pairs $\left(f_{\lambda}, E_{\lambda}\right)$ are also extremal. Notice that $f_{\lambda}$ has a critical value $a+\lambda$, so taking $\lambda=\delta a / 2$ we obtain an extremal pair where the polynomial has critical value $a(1+\delta / 2)$.

By iterating this construction, we obtain a sequence of extremal pairs $\left(f_{k}, E_{k}\right)$, where for each $k$ the polynomial $f_{k}$ has a critical value $a(1+\delta / 2)^{k}$.

We can normalize each $f_{k}$ and $E_{k}$ as in (5), so that the family $\left\{f_{k}\right\}$ will be uniformly bounded on compact subsets of $\mathbf{C}$, and we will obtain a limit pair $\left(f_{\infty}, E_{\infty}\right)$, where $f_{\infty}$ evidently has smaller degree than $f$. From (10) we conclude that

$$
\operatorname{cap} E \sup _{E}\left|f^{\prime}\right|=\operatorname{cap} E_{\infty} \sup _{E_{\infty}}\left|f_{\infty}^{\prime}\right| \text {. }
$$

Now we use induction on $d$. Denote the left-hand side of (4) by $C(d)$. Evidently $C(1)=1$. So Theorem 2 holds with $d=1$. Suppose that $k>1$ is the smallest integer for which the inequality of Theorem 2 does not hold with $d=k$. Applying Lemma 1 with $d=k$ we obtain an extremal pair $(f, E)$, where $\operatorname{deg} f=k$, because the right-hand side of (3) is strictly increasing. Then

$$
C(k)>C(k-1),
$$

again because the right-hand side of (3) is strictly increasing. If $E(f)$ is connected, then we use Theorem A to conclude that Theorem 2 holds with $d=k$, contrary to our assumption.

If $E(f)$ is disconnected we obtain a contradiction between (11) and (12), because $\operatorname{deg} f_{\infty}<k$ and Theorem 2 holds for all $d<k$. This contradiction shows that the number $k$ does not exist, so Theorem 2 holds for all $d$.

It remains to address the case of equality. We already saw in the course of the proof that equality cannot occur when $E(f)$ is disconnected. This reduces the case of equality in Theorem 2 to the case of equality in Theorem A.

This paper was written when the author was visiting Christian-Albrechts University of Kiel. The author thanks Vladimir Andrievski and Walter Bergweiler for stimulating discussions and the referee for suggestions that improved the exposition.

\section{REFERENCES}

[1] L. Ahlfors, Lectures on quasiconformal mappings, Van Nostrand, Toronto 1966. MR0200442 $(34: 336)$

[2] L. Ahlfors, Conformal Invariants. Topics in geometric function theory, McGraw-Hill, NY 1973. MR0357743 (50:10211)

[3] A. Douady, Prolongement de movements holomorphes, Sém. Bourbaki 1993/4. Astérisques 227 (1995) Exp. No. 775 7-20. MR1321641 (95m:58104)

[4] A. Eremenko and G. Levin, Estimates of the characteristic exponents of a polynomial. (Russian) Teor. Funkt. Funktsional. Anal. i Prilozhen. No. 58, 30-40 (1993); English transl. J. Math. Sci. (New York) 85 (1997) no. 5 2164-2171. MR.1258059(95e:30027)

[5] A. Eremenko and L. Lempert, An extremal problem for polynomials. Proc. AMS 122 (1994) 191-193. MR1207536 (94k:30007) 
[6] A. Eremenko and W. Hayman, On the length of lemniscates, Michigan Math. J. 46 (1999) 409-415. MR.1704189 (2000k:30001)

[7] A.A. Markov, On a question of Mendeleiev, Petersb. Abhandl. LXII (1890) 1-24.

[8] Q. Rahman and G. Schmeisser, Les inégalités de Markoff et de Bernstein, Sém. Math. Supér., Presses de l'Université de Montréal, Montréal, QC, 1983. MR0729316 (85f:41009)

[9] G. Pólya, How to solve it? Princeton UP, Princeton NJ 1945. MR0011666 (6:198f)

[10] Ch. Pommerenke, On the derivative of a polynomial, Michigan Math. J. 6 (1959) 373-375. MR0109208 (22:95)

Department of Mathematics, Purdue University, West Lafayette, Indiana 47907

E-mail address: eremenko@math.purdue.edu 